\title{
Shape Characterization of Atrial Fibrillation using Time-Frequency Analysis
}

\author{
M Stridh, L Sörnmo \\ Dept. of Electroscience, Lund University, Sweden
}

\begin{abstract}
A new method for characterization of atrial fibrillation in the surface ECG is presented which is based on a time-frequency distribution of the QRST-cancelled signal. With the present method, information regarding temporal variations in fibrillation frequency and waveform shape is extracted and investigated. The method is evaluated on a database including 182 recordings (obtained during resting conditions) from patients diagnosed with atrial fibrillation. The results show that a high fibrillation frequency often is associated with a sinusoidal shape whereas a low frequency is more associated with a sawtooth-like shape.
\end{abstract}

\section{Introduction}

ECG-based characterization of atrial fibrillation has mainly been done in terms of the average or dominant fibrillation frequency (or repetition rate) which serves as an index of atrial organization [1], [2]. In these studies, spectral analysis techniques are used to estimate the dominant repetition rate by means of the maximum peak location, the variation in rate by the width of the peak and the average signal energy by the peak amplitude. This method is relatively simple but provides valuable clinical information.

Techniques for a more detailed characterization of atrial fibrillation has been suggested with which a second-tosecond resolution of the fibrillation frequency can be achieved [3]. This study revealed large and rapid variations in the repetition rate over only a few seconds. Different strategies were therefore suggested for tracking of these short-term variations and for tracking of small amplitude long-term changes caused by different interventions. In both cases, our spatiotemporal QRST cancellation method was used for suppression of the ventricular activity in the surface ECG [4].

In the present work, we have developed a new algorithm for frequency estimation which uses the shape of the patient's individual fibrillatory waveform in order to achieve better and more robust estimates of the fibrillation frequency. The suggested algorithm sequentially parameterizes the residual ECG signal into frequency, amplitude and shape parameters with a second-to-second resolution. The method is well-suited for real-time applications.

\section{Material and acquisition}

The method was evaluated on a database including 182 recordings (obtained during resting conditions) from patients diagnosed with atrial fibrillation (and in which a repetitive atrial signal was discernable). Each recording was of one minute duration and only lead $V_{1}$ was investigated (although $V_{2}$ and $V_{3}$ were also used for QRST cancellation). All recordings were acquired using equipment by SiemensElema AB, Sweden. The ECG's were sampled at $1 \mathrm{kHz}$ with an amplitude resolution of $0.6 \mu \mathrm{V}$.

\section{Methods}

The present method is based on the assumption that atrial fibrillation signals locally (over 1-2 seconds) are close to periodic, i.e., can be described by a fundamental frequency corresponding to the rate of the signal waveform and a harmonic pattern describing the shape. Further, it is assumed that the shape (normalized to a certain rate and amplitude) varies slowly over time while the rate and amplitude of the shape vary over a few seconds. These assumptions should also be adequate for other atrial conditions such as atrial flutter and atrial tachycardia which makes the usefulness of the algorithm broader.

\section{1. $\quad$ Preprocessing}

All signals were filtered in order to remove baseline wander. Detection and classification of the QRS complexes were performed before spatiotemporal QRST cancellation was applied [4]. Since the spectral content of interest in the resulting residual ECG signal is well below $25 \mathrm{~Hz}$, the residual ECG is downsampled from $1 \mathrm{kHz}$ to $50 \mathrm{~Hz}$ which thus substantially reduces the amount of data to be processed. In Fig. 1, two examples of ECG signals from two different patients with atrial fibrillation are shown. The first panel shows a more organized fibrillation signal before QRST cancellation and the second panel shows the same signal after QRST cancellation. The third and fourth panel 
show a less organized fibrillation signal before and after QRST cancellation.

(a)

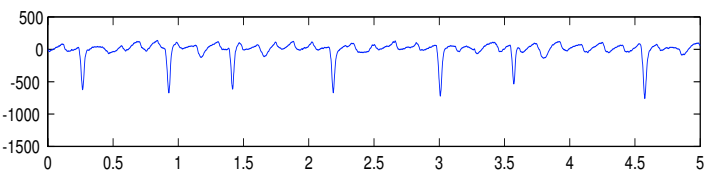

(b)

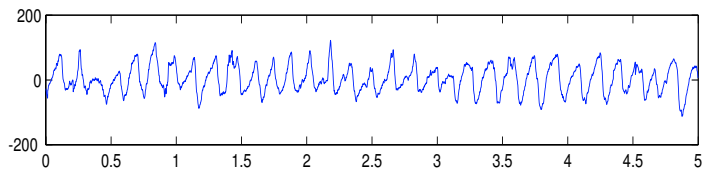

(c)



(d)

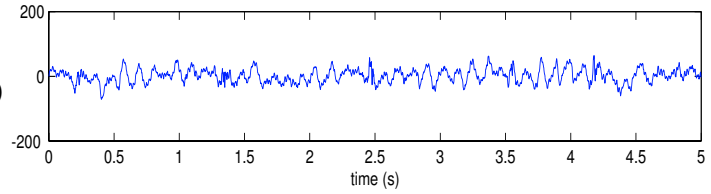

Figure 1. Preprocessing of two 5 seconds examples of atrial fibrillation: More organized case is shown in (a) before and (b) after QRST cancellation, and a less organized case is shown in (c) before and (d) after QRST cancellation.

\subsection{Shape characterization}

For every new block containing $L=50$ samples of the preprocessed residual ECG, the shape characterization method transforms the $K=128$ most recent samples, represented by the vector $\mathbf{x}_{k}$, into the frequency domain creating a new spectrum, $\mathbf{q}_{k}$. The frequency transformation is done using a nonuniform DFT (represented by the DFT matrix F) with a logarithmic frequency scale $(2.5-25 \mathrm{~Hz})$ such that two harmonic spectra with different fundamentals can be aligned to each other with both the fundamentals and all the harmonics still matching.

$$
\mathbf{q}_{k}=\mathbf{F W} \mathbf{x}_{k}
$$

The diagonal matrix $\mathbf{W}$ is a Hamming window applied to the input signal.

The nonuniform spectrum, $\mathbf{q}_{k}$, is modelled as a scaled $\left(a_{k}\right)$ and frequency-shifted $\left(\theta_{k}\right)$ version of a spectral profile, $\tilde{\phi}_{k}$, which is a function representing the shape of the atrial signal waveform.

$$
\mathbf{q}_{k}=a_{k} \mathbf{J}_{\theta_{k}} \tilde{\boldsymbol{\phi}}_{k}, \quad k=1,2, \ldots
$$

The notation $(\tilde{\cdot})$ denotes that the vector is pre- and appended with $\Theta$ (the maximum number of frequency shift) samples in order to allow that different parts of $\tilde{\phi}_{k}$ can be selected.
Weighted least-squares estimation of the frequency shift and amplitude is performed using the following cost function

$$
J\left(\theta_{k}, a_{k}\right)=\left(\mathbf{q}_{k}-a_{k} \mathbf{J}_{\theta_{k}} \tilde{\boldsymbol{\phi}}_{k}\right)^{T} \mathbf{D}\left(\mathbf{q}_{k}-a_{k} \mathbf{J}_{\theta_{k}} \tilde{\boldsymbol{\phi}}_{k}\right)
$$

where the weight matrix $\mathbf{D}$ is used to compensate for the oversampling at low frequencies associated with a logarithmic frequency scale.

I nitially, the spectral profile is simply a peak at a certain reference position (e.g. $5 \mathrm{~Hz}$ ). Then, the spectral profile is updated with each new spectrum. The important difference compared to when calculating an average spectrum is that the spectral profile is always updated with the fundamental of the new spectrum aligned to the reference frequency which makes the spectral profile independent of the location of the fundamental and the variation around it. The spectral profile thus only represents the overall shape and is therefore much less smeared than an ordinary average spectrum. Two decomposed time-frequency distributions are shown in Fig. 2a. The spectral profiles compared to the broader average spectra are shown in Fig. 2b and in Fig. 2c the resulting frequency trends are shown.
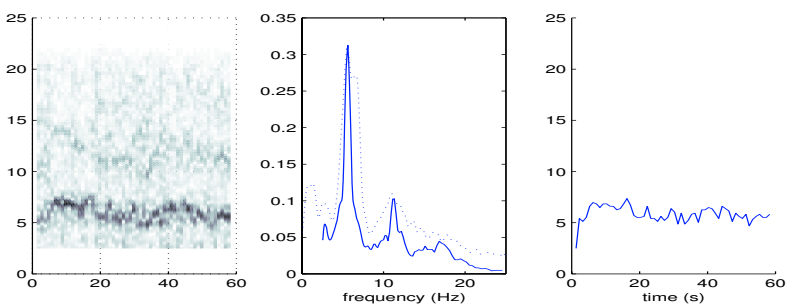

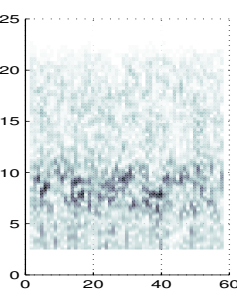

(a)

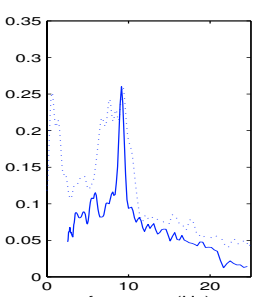

(b)

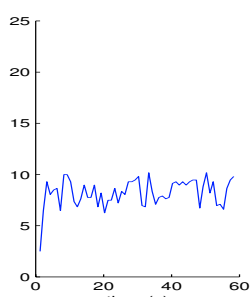

(c)
Figure 2. Decomposition of two different 1 minute time-frequency representations: In (a), the time-frequency distributions of the atrial fibrillation signals are shown, in (b), the spectral profiles (solid) and power spectrum of the entire minute (dotted) are plotted and in (c), frequency trends are shown.

After updating the spectral profile, it is mapped into two shape parameters, $b_{k}$, which is the amplitude of the fundamental in the normalized spectrum (a low value indicates a smeared spectrum), and $\gamma_{k}$, that describes the exponential decay of the harmonic spectrum; a more sawtooth-shaped atrial activity has relatively large 
harmonics resulting in a low exponential decay while a more sinusoidal shape has very low or no harmonics resulting in a higher exponential decay. The fundamental and the harmonics are located at the following positions in the spectral profile

$$
P(i)=p_{k}+K\left\lfloor\log _{10}(i+1)\right\rfloor
$$

where $p_{k}$ is the position of the fundamental. The peak amplitudes at these positions in the spectral profile are, using least-squares estimation, matched to the exponential model

$$
\phi_{k}(P(i))=b_{k} e^{-\gamma_{k} i} \quad i=0,1, \ldots, m
$$

resulting in the following parameter estimates

$$
\begin{aligned}
& \hat{b}_{k}=e^{\frac{2(2 N-1)}{M^{2}+M} \sum_{i=0}^{m} \ln \phi_{k}(P(i))-\frac{6}{M^{2}+M} \sum_{i=0}^{m} i \ln \phi_{k}(P(i))} \\
& \hat{\gamma}_{k}=-\frac{6}{M^{2}+M} \sum_{i=0}^{m} \ln \phi_{k}(P(i))+\frac{12}{M^{3}-M} \sum_{i=0}^{m} i \ln \phi_{k}(P(i))
\end{aligned}
$$

Athough $b_{k}$ is an important parameter containing information about the smearing in the spectral profile it is not considered in this study.

A important issue is to continuously detect if an atrial signal is present. This can be done using a signal-tonoise ratio, $\kappa_{k}$, defined as the relation between the signal amplitude, which here is the average amplitude of the fundamental and the first harmonic, and the noise level in between these two peaks,

$$
\kappa_{k}=\frac{\phi_{k}\left(p_{k}\right)+\phi_{k}\left(p_{k}+\left|\log _{10} 2\right|\right)}{2 \phi_{k}\left(p_{k}+\left|\log _{10} 1.5\right|\right)} .
$$

\section{Shape simulation}

The suggested model can represent a large number of different repetitive signals ranging from sinusoidal to pulse-shaped biphasic waveforms over more sawtooth-like shapes, see Fig. 3. The pulse-shaped biphasic shapes have large harmonics representing the sharp transitions of these signals and therefore have a low exponential decay of the harmonic amplitudes, typically around 0.25 0.5. Sawtooth-like shapes have smaller but still distinct harmonics resulting in an exponential decay around one. Finally, more sinusoidal signals have very low or no discernable harmonics resulting in an exponential decay as high as two. It should be noted that the sharpness of different shapes resulting in the harmonics is normalized to both amplitude and frequency. Thus, a sawtooth-shaped signal at $6 \mathrm{~Hz}$ can in fact have sharper edges than a pulseshaped biphasic shape at $1 \mathrm{~Hz}$.

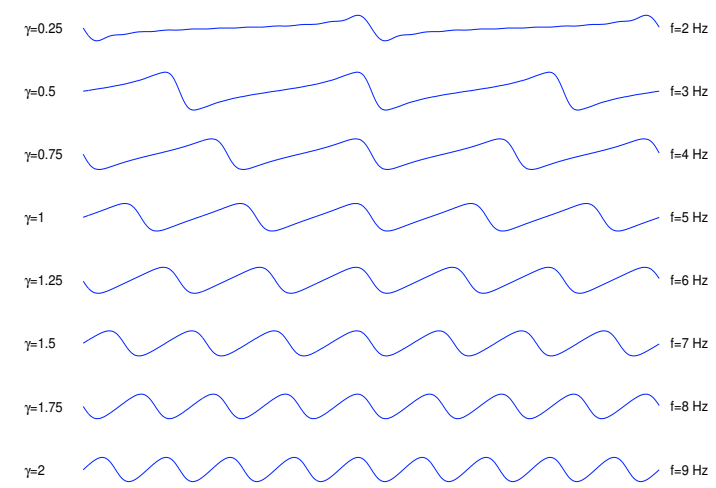

Figure 3. Simulation study of the exponentially decaying spectral profile model. From the top with increasing exponential decay and increasing frequency

\section{Results}

The exponential decay is illustrated in Fig. 4, where the spectral profiles for the two cases are parameterized resulting in different exponential decays. The more organized fibrillation has an exponential decay of 1.0 indicating a sawtooth-like shape while the less organized case has an exponential decay of 1.8 corresponding to a sinusoidal shape.

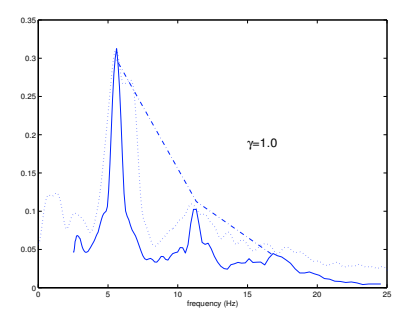

(a)

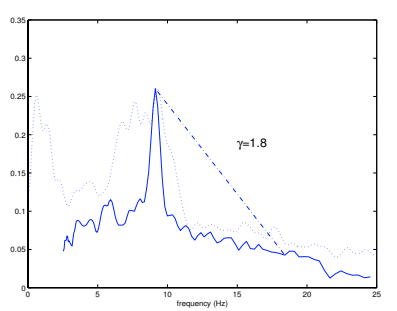

(b)
Figure 4. Spectral profile (solid), power spectrum of the entire minute (dotted) and exponential decay model (dashdotted) for (a) more organized case and (b) less organized case.

The frequency, amplitude, exponential decay and signalto-noise ratio is used to characterize the atrial signal on a second-to second basis. Fig. 5 shows one minute trends of each of these parameters for the more organized of the two cases. It is noted that the frequency estimate is immediately found despite that the spectal profile more slowly tunes in the correct shape (exponential decay). A mentioned above, this example tunes in at an exponential decay of around one indicating a sawtooth-shaped signal. The initial shape tuning can be done arbitrarily fast. However, a quite low adaptation gain is used in order to robustify the shape tracking performance resulting in a tuned shape after about $30 \mathrm{~s}$. The signal-to-noise ratio, $\kappa_{k}$, is calculated based on 
the spectral profile and therefore it takes the same time as does the exponential decay. In this study, only signals with a signal-to-noise ratio of above 2.6 were considered. For lower values, no atrial signal was discernable in the residual ECG. For the case in Fig 5d, the signal-to-noise ratio is around 7 indicating an average of the fundamental and first harmonic amplitudes around seven times the amplitude in between.

(a)

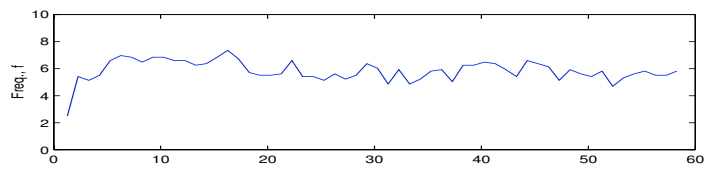

(b)



(c)

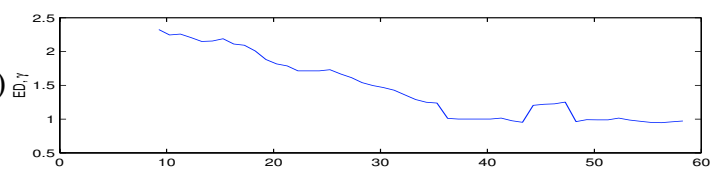

(d)

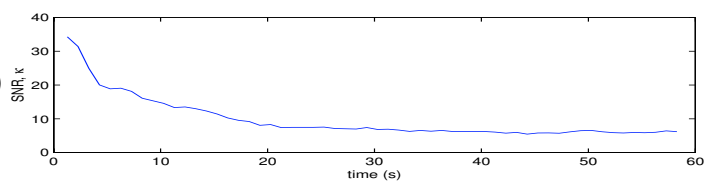

Figure 5. Parameter trends: (a) frequency, (b) amplitude, (c) exponential decay and (d) signal-to-noise ratio.

Each of the 182 patients were placed into a frequencyshape space resulting in Fig. 6 based on the average frequency and average exponential decay during the last 20 seconds of the one minute segment. Rapid and sinusodal shapes are placed in the upper right corner while slower and more pulse-shaped signals are placed in the lower left corner. In between, there are more sawtooth-shaped signals. Fig. 6 shows that a large correlation exists between higher frequencies and more sinusoidal shape and lower frequencies and a more sawtooth-like or pulse-shaped signals. The arrow indicates the direction from more to less organized atrial signals.

\section{Conclusion}

The results show that each spectrum is well-modeled by an amplitude-scaled and frequency-shifted spectral profile. Further, it is found that the method always produces narrower spectral peaks, i.e., fundamental and one or several harmonics, when compared to the conventional power spectrum. An interesting finding is that there is a strong correlation between fibrillation frequency and wave shape: a high frequency is often associated with a more



Figure 6. Evaluation database: Frequency-shape space

sinusoidal shape whereas a low frequency is associated with a more sawtooth-like shape.

\section{References}

[1] Holm M, Pehrsson S, Ingemansson M, Sörnmo L, J ohansson R, Sandhall L, Sunemark M, Smideberg B, Olsson C, Olsson B. Non-invasive assessment of atrial refractoriness during atrial fibrillation in man - introducing, validating and illustrating a new ECG method. Cardiovasc Res 1998 ;38 :69_ 81.

[2] Bollmann A, K anuru N, McTeague K, Walter P, DeLurgio D, Langberg $\mathbf{J}$. Frequency analysis of human atrial fibrillation using the surface electrocardiogram and its response to ibutilide. Ameri J Cardiol J une $1998 ; 8$ 1:1439-1445.

[3] Stridh M, Sörnmo L, Meurling CJ, Olsson SB. Characterization of atrial fibrillation using the surface ECG: Time-dependent spectral properties. IEEE Trans Biomed Eng 2001;48 (1):19-2 7.

[4] Stridh M, Sörnmo L. Spatiotemporal QRST cancellation techniques for analysis of atrial fibrillation. IEEE Trans Biomed Eng 2001;48 (1):105-111.

Address for correspondence:

Martin Stridh

Dept. of Electroscience

Lund University

P.O. Box 118

SE-2 2100 Lund

martin.stridh@es.lth.se 